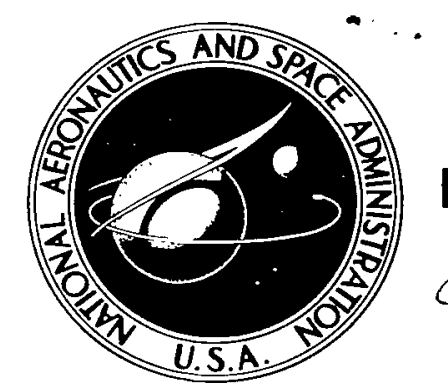

NASA TN D-2992

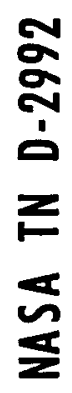
(i. 1

\title{
THERMAL ESCAPE OF
}

NEUTRAL HYDROGEN AND

ITS DISTRIBUTION IN

THE EARTH'S THERMOSPHERE

by Mordehai Liwshitz and S. F. Singer

NATIONAL AERONAUTICS AND SPACE ADMINISTRATION - WASHINGTON, D.

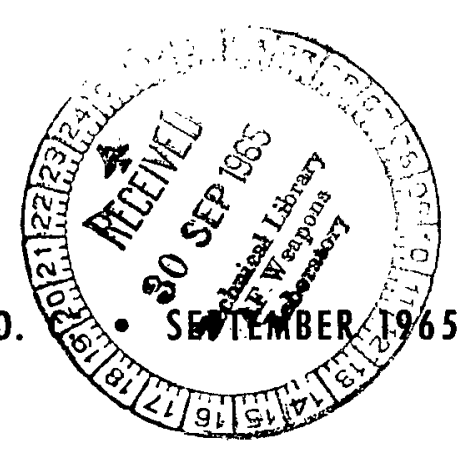


NASA TN D-2992

\section{THERMAL ESCAPE OF NEUTRAL HYDROGEN AND ITS DISTRIBUTION IN THE EARTH'S}

THERMOSPHERE

By Mordehai Liwshitz

Goddard Space Flight Center

Greenbelt, Md.

and

S. F. Singer

University of Miami

Coral Gables, Fla. 


\title{
THERMAL ESCAPE OF NEUTRAL HYDROGEN AND ITS DISTRIBUTION IN THE EARTH'S THERMOSPHERE
}

\author{
by \\ Mordehai Liwshitz \\ Goddard Space Flight Center \\ and \\ S. F. Singer \\ University of Miami
}

\begin{abstract}
SUMMARY
In this paper the effect of thermal escape on the distribution of neutral hydrogen in the upper thermosphere is discussed. A simplified model is proposed which assumes an extended transition region between the collision dominated lower thermosphere and the effectively collisionless exosphere. In this region collisions with the predominant constituent, oxygen, are still too frequent to be neglected, while the effect of thermal escape is strong enough to impede the approach to local thermal equilibrium. The behavior of hydrogen in the transition region is investigated for different oxygen temperatures by means of the Monte Carlo method. Results show that stationary conditions are attained in relatively short times which vary from about one hour at $1500^{\circ} \mathrm{K}$ to about one-half hour at $2500^{\circ} \mathrm{K}$. The velocity distribution of hydrogen is seriously perturbed, and shows marked anisotropy as a result of escape; consequently, the loss rate of neutral hydrogen from the terrestrial atmosphere may be reduced by a factor of three or more in the range of temperatures considered.
\end{abstract}




\section{CONTENTS}

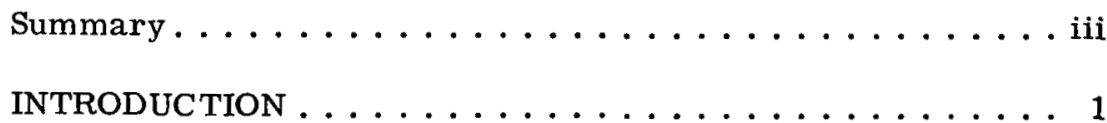

THE CONVENTIONAL APPROACH TO THE PROBLEM AND A NEW SIMPLIFIED MODEL . . . . . . . . . . . 2

SOLUTION OF THE TRANSPORT PROBLEM BY MEANS

OF THE MONTE CARLO METHOD .............6

RESULTS OF THE MONTE CARLO COMPUTATION . . . . . 11

ACKNOWLEDGMENTS . . . . . . . . . . . . . 23

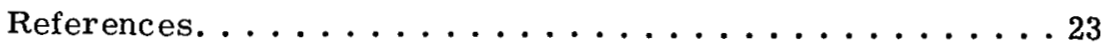




\title{
THERMAL ESCAPE OF NEUTRAL HYDROGEN AND ITS DISTRIBUTION IN THE EARTH'S THERMOSPHERE
}

\author{
by \\ Mordehai Liwshitz \\ Goddard Space Flight Center \\ and \\ S. F. Singer \\ University of Miami
}

\section{INTRODUCTION}

With the realization that a real gas at finite temperatures is actually an assembly of a vast number of particles moving at random velocities, it was recognized that planetary atmospheres may be subject to selective thermal escape. This mechanism favors the light elements on account of their higher thermal velocities. However, since the early inception of this problem the quantitative treatment of the effect has not advanced greatly beyond the original crude estimates of atmospheric escape rates (References $1,2,3$, and 4).

The exploration of space in recent years has renewed and expanded the interest in the subject of atmospheric escape. Proper understanding of either the processes involved in the formation of the earth's radiation belts, or a clearer insight into the phenomenon of night-sky Lyman- $\alpha$ radiation, requires a more precise knowledge of conditions in the earth's exosphere. Beyond an altitude of roughly $1000 \mathrm{~km}$ (depending on thermal conditions), the primary constituents of the exosphere are now assumed to be the light elements helium and hydrogen. These are precisely the elements whose distribution may be subject to effects of escape from the earth's gravitational field. It is the purpose of the present paper to investigate in greater detail than attempted hitherto the effects on the distribution of hydrogen just below, and at the base of, the exosphere, and to present results which, hopefully, are closer to the actual physical situation than those now widely used in applications to problems of atmospheric physics.

As we see it, the core of the problem lies in the non-equilibrium situation with which escape confronts us. As we shall demonstrate in the next section, the well known escape formula of Jeans (Reference 4) never holds rigorously, and the effects of the inconsistency appear to have been underestimated in the past. At best, escape rates based on this formula represent upper limits to the actual loss, while quantities which are sensitive to the actual velocity distribution of hydrogen at the base of the exosphere may be more seriously affected. A typical example is the concentration 
of hydrogen at large geocentric distances (Reference 5). Whereas previous attempts at solving the problem of the hydrogen velocity distribution near the critical level have concentrated on the high energy tail (References 6, 7, 8, and 9), our treatment covers all velocity ranges (Reference 10).

For the purpose of this investigation a simple model (suggested by the physical situation) was constructed of a semi-infinite container with a semi-permeable upper boundary. Within the framework of this model, the problem was treated by means of the Monte Carlo method, which appears appropriate in view of its basic stochastic character.

As a first step in attacking the problem, we considered the establishment of a quasi-steady state in a layer a few mean free paths in depth extending below the assumed base of the exosphere; that is, we assumed the temperature of the oxygen environment, in which hydrogen is embedded at such altitudes, to be held constant long enough for hydrogen to approach a steady state and obey the equation of continuity. Strong emphasis was placed on consistency of the results with the underlying assumptions.

Results of our computations lend support to the concept of a moderately sharp transition to an essentially collisionless exosphere. There is, indeed, a critical region in the atmosphere, just below the assumed base of the exosphere, where departure from a collision-dominated situation may be observed. It is, however, precisely in this region, where effects of escape compete with collisions in shaping the distribution of hydrogen. Furthermore, the fact should not be overlooked that the altitude of this critical level shifts considerably as a result of the thermal expansion and contraction of the main atmosphere. Consequently, the lower boundary of the exosphere will depart to some extent from spherical symmetry.

Results also bear out the earlier estimate (Reference 10) that the escape rate will be reduced considerably, and that the whole velocity distribution will depart from the equilibrium distribution appropriate to the ambient atmospheric temperature. The process ensuing from escape may best be described as anisotropic cooling of hydrogen, which entails both the anisotropy and reduced dispersion of velocities resulting from the rapid loss of fast particles.

Let us now proceed to a critical review of the conventional approach and search for new avenues to a better understanding, suggested by our examination.

\section{THE CONVENTIONAL APPROACH TO THE PROBLEM AND A NEW SIMPLIFIED MODEL}

The escape parameter $\mathrm{E}$ at distance $\mathrm{R}$ may be expressed by

$$
E=\frac{m v_{\infty}^{2}}{2 k T}
$$

where

$$
\begin{aligned}
& \mathrm{R}=\text { geocentric distance of the escape level }(\mathrm{cm}), \\
& \mathrm{m}=\text { mass of hydrogen atom (grams) }
\end{aligned}
$$




$$
\begin{aligned}
& \mathrm{v}_{\infty}=\text { escape velocity of a hydrogen atom }(\mathrm{cm} / \mathrm{sec}), \\
& \mathrm{k}=\text { Boltzmann's constant }\left(1.38 \cdot 10^{-23} \text { joules } /{ }^{\circ} \mathrm{K}\right), \\
& \mathrm{T}=\text { temperature at escape level }\left({ }^{\circ} \mathrm{K}\right) .
\end{aligned}
$$

The Jeans escape formula may then be written in terms of the escape parameter, E, as

$$
F_{e s}=4 \pi R^{2} N_{0}\left(\frac{k T}{2 \pi m}\right)^{\frac{1}{2}}(1+E) e^{-E}
$$

where

$$
\mathrm{N}_{0}=\text { hydrogen concentration at escape level (atom } / \mathrm{cm}^{3} \text { ). }
$$

The Jeans escape formula is obtained by multiplying the velocity distribution,

$$
f(R, v, \theta, \phi)=\left(\frac{m}{2 \pi k T}\right)^{3 / 2} e^{-m v^{2} / 2 k T} v^{2} \sin \theta
$$

by $\mathrm{v} \cos \theta$ and integrating over $\mathrm{v}_{\infty} \leq \mathrm{v}<\infty, 0 \leq \theta \leq \pi / 2,0 \leq \phi \leq 2 \pi$, and over the spherical surface at $\mathrm{R}$ as well, where

$$
v \text { = velocity of hydrogen atom }
$$

and

$$
\theta \text { and } \phi=\text { polar angles in velocity space. }
$$

But Equation 1 cannot be exact from a physical point of view because an escape flux implies a net flux, and the gas possesses a flow velocity $\vec{w}$ in the radial direction (Reference 11). Even if $\vec{w}$ were unifor $m$, the velocity distribution in the frame of the planet would obviously be proportional to

$$
\exp \left[-\frac{m(v-w)^{2}}{2 k T}\right]=\exp \left[-\frac{m\left(v^{2}+w^{2}+2 v w \cos \theta\right)}{2 k T}\right]
$$

and therefore isotropy is destroyed. While in this frame the above limits of integration are the correct ones, the proper results of integration would differ from Equation 1, in view of Equation 3. If, on the other hand, we chose to integrate in the moving frame, as is effectively done by putting Equation 2 in the integrand, we should have multiplied the velocity distribution $f$ by $(v \cos \theta)-w$, and changed the limits of integration, both over $v$ and $\theta$, from $v$ to $(v-w)^{2}$ and from $\pi / 2$ to $\cos ^{-1}(-w / v)$, respectively. However, Equation 1 may be considered a fair approximation provided $w \ll v$ (implying $E \gg 1$ ), and provided $v^{2} e^{-\beta v^{2}}$ tends to go to zero rapidly enough. This implies a highly peaked velocity distribution (i.e., a low temperature for a Maxwellian) which contradicts somewhat the requirement that $w \ll v$ for most values of $v$. This appears to be one of the reasons for the ready acceptance of Jeans' formula, at least by workers in terrestrial aeronomy. Until quite recently it was not realized that thermospheric temperatures may go as high as 2000$2500^{\circ} \mathrm{K}$, or even higher for short periods of time. 
Acceptance of Equation 1 implies, moreover, another assumption about the form of the velocity distribution at the base of the exosphere, namely that

$$
\mathrm{f}=0 \quad \text { where } \quad-\infty<\mathrm{v} \cos \theta<-\mathrm{v}_{\infty}
$$

and

$$
\mathrm{f}=\mathrm{fm} \quad \text { where } \quad-\mathrm{v}_{\infty}<\mathrm{v} \cos \theta<\mathrm{v}_{\infty}
$$

where $\mathrm{f}_{\mathrm{m}}$ is the Maxwellian distribution, Equation 2. Then, however, the normalization constant used $(\mathrm{m} / 2 \pi \mathrm{kT})^{3 / 2}$, is no longer strictly valid, again with the result that Equation 1 is no longer exact.

We have gone into the detailed formal discussion of the simple Equation 1 for two main reasons: First it points out the limitations of this formula's applicability, and secondly the conditions expressed by Equation 4 may serve as guidelines for further attempts at a solution. Indeed, if one accepts the model of a sharp transition to a collisionless exosphere, one may obtain a set of plausible boundary conditions for $f$ at $R$ by relaxing the requirement that $f$ be Maxwellian in the interval $\left(-v_{\infty}, v_{\infty}\right)$. As we shall see below, the results of our model computations agree very well with these assumptions.

We shall not go here into a discussion of further difficulties involved in Equation 1 and attempts to resolve them, as this has been done by one of the authors elsewhere (Reference 12); instead, we proceed directly to a brief exposition of our simplified model and the method applied in its solution.

In consequence of the above discussion the pertinent facts in relation to the escape problem appear to be that equilibrium is effectively destroyed by thermal evaporation of the escaping element and that near-equilibrium theories, such as are implied by the application of Chapman's diffusion equation (References 13 and 14), do not yield the desired information about the actual velocity distribution of the escaping element close to the base of the exosphere.

If one considers, however, some time scales which arise naturally in the context of the escape problem, a considerable simplification in the treatment of the problem may be achieved. The two significant times we have in mind are effusion time, $t_{e}$, and diffusion time, $t_{d}$, to be defined as follows.

Let the base of the exosphere be at altitude $z_{0}$, and the diffusion velocity of the escaping element at altitude $z^{\prime}$ be $w\left(z^{\prime}\right)$. Since $w\left(z^{\prime}\right)=\overline{d z^{\prime} / d t}$, an average flow velocity (directed upwards in the case of hydrogen or helium), the mean time of transport for hydrogen (or helium) from any arbitrary altitude $z<z_{0}$, to the base of the exosphere is given by

$$
t_{d}(z)=\int_{t(z)}^{t\left(z_{0}\right)} d t=\int_{z}^{z_{0}} \frac{d z^{\prime}}{w\left(z^{\prime}\right)} .
$$


Taking the difference between, say $t_{d}\left(z_{1}\right)$ and $t_{d}\left(z_{2}\right)$ one obtains, in effect, the time of sojourn of a diffusing ensemble between two levels $z_{1}$ and $z_{2}$. Of course, w( $z$ ) has to be obtained from a solution of the problem, but we may use as an ad hoc approximation a formula such as Equation 12 in Reference 14.

The definition of effusion time, $t_{e}$, is based on the consideration that since in reality escape does not take place from a sharply defined layer, it is reasonable to state that the bulk of escaping hydrogen (or helium) will be derived from a layer on the order of an atmospheric scale height, below the exosphere, and that in the escape region the mean free path is of about equal dimensions. Atoms diffusing from below this layer have a high probability of colliding in it and atoms above it are likely to have had their last collision there. Consider now the thermal evaporation of such a layer in the absence of sources. This process is governed by the rate equation

$$
\frac{\mathrm{d} \eta}{\mathrm{dt}}=-\frac{\eta}{\mathrm{t}_{\mathrm{e}}}
$$

where $\eta \mathrm{cm}^{-2}$ is the content per $\mathrm{cm}^{2}$ of such a layer, given by $\eta=\mathrm{NH}$, where $\mathrm{N}$ and $\mathrm{H}$ are some mean values of the concentration and scale height in this layer.

On the other hand, $\mathrm{d} \eta / \mathrm{dt}$, is the instantaneous escape flux, given (ad hoc) by Equation 1, whence

$$
t_{e}=H\left(\frac{2 m}{k T}\right)^{1 / 2}(1-E) e^{E}
$$

It appears that the ratio $t_{d} / t_{e}$ may serve as a reasonable criterion in estimating the departure of the distribution from equilibrium. Once a set of conditions is reached where this ratio dwindles below unity, escape and diffusion will be of comparable significance in shaping the distribution of the escaping element. It suggests that we pay special attention to this limited critical region, and furthermore implies a subdivision of the upper atmosphere (above the turbopause) into three main regions:

1. The bulk of the thermosphere, where diffusion theory according to Chapman appears entirely adequate (Reference 14).

2. The exosphere, where orbital theory (Reference 11), use of Liouville's equation (Reference 15), and the collisionless Boltzmann equation (Reference 16) yield equivalent results.

3. Between these regions an extended transition region of a few mean-free-paths depth. Here, the approaches used in the other regions fail.

From the nature of the criteria chosen it should be evident that the boundaries of the transition region are not sharply defined. But in analogy with the theory of the exosphere it appears reasonable to postulate sharp boundaries, provided sufficient care is exercised in their choice. Our model will be plausible and internally consistent if, within certain limits, the results are not sensitive to the particular boundaries chosen. This assures us that the characteristic structure of our results is not a spurious boundary effect, but results from intrinsic properties of the physical processes investigated in our model. How this can be achieved with confidence in our results will be shown later. 
The fact that all throughout the transition region hydrogen is a minor constituent with a relative concentration of less than $3 \times 10^{-3}$ justifies further essential simplification in our model (i.e., the neglect of hydrogen-hydrogen interaction). Changes in the hydrogen distribution evolve from interaction with the surrounding oxygen medium, which constitutes an essentially undisturbed thermal bath.

A crucial simplification results from a consideration of the trajectories followed by atoms in the exosphere: those with sufficient velocity are lost "forever"; those below escape energy, however, describe ballistic orbits. In spherical symmetry, we may then assume that for each particle in this class leaving the escape level, a corresponding mirror particle enters from the exosphere after a certain time delay. This suggests that the effect of ballistic orbits may be simulated by the boundary conditions at the top: the escape level may be regarded as a selectively permeable membrane. Particles above escape energy pass through it unhindered, while slower atoms may be considered to be specularly reflected with an appropriate time delay. Once a steady state has been approached, of course, there is no time delay and outgoing atoms are exactly balanced by incoming particles.

This property becomes especially simple, mathematically, when in view of the small thickness of the transition region a plane-parallel approximation is adopted. The nature of the boundary conditions at the top is very similar to those of Equation 4. The main distinction, however, is that no particular form of the distribution function can be postulated, as was done there. All that one can observe is that near the boundary the distribution function should be symmetrical in the vertical component of velocity, $v_{z}$, in the domain $\left(-v_{\infty}, v_{\infty}\right)$.

Finally the fact that particles are fed into the region of interest from below by diffusion can be taken into account by postulating a source, located at the lower boundary, and (in the steady state case) injecting particles at a constant rate. The actual form of the source flux is of no great import, however, since in the lower portion of the transition region collisions are still frequent enough to bring about a fair amount of randomization. Our results bear out the contention that apart from a region in the vicinity of the source, the steady state distribution is not sensitive to the particular form of the source flux.

The considerations expressed suggest the simple physical model we were searching for: a container, extending infinitely in the $x-y$ directions and of finite height in $z$. The container is filled with a major component gas in thermal and diffusive equilibrium, subject to a spatial distribution determined by gravity and temperature alone. After injection by a source at the bottom, atoms of the minor constituent percolate through this medium until they reach the semipermeable top of the container where (depending on their velocity) they may be either reflected or pass through.

\section{SOLUTION OF THE TRANSPORT PROBLEM BY MEANS OF THE MONTE CARLO METHOD}

Though the model just delineated appears rather simple and well defined, solution of the corresponding transport equation (the linear Boltzmann equation) by analytical methods is most 
difficult without surrendering much of its physical content by simplifying approximations. The main difficulty lies in the highly singular boundary conditions such as the tripartite division of the velocity range in the vicinity of the escape level $(-\infty,-v \infty),\left(-v^{\infty}, v^{\infty}\right),\left(v^{\infty}, \infty\right)$, and the finite extent of the vertical dimension. The small depth of the container (representing the transition region) indicates, however, that the Monte Carlo method may be applied with a good chance of success.

A previous Monte Carlo treatment of hydrogen diffusion (Reference 17) did not take into account escape, and, with its implicit use of the Maxwell-Boltzmann distribution for hydrogen, did not aim at elucidating the question of the velocity distribution near the escape level. In distinction to that treatment which involved an optical model of the atmosphere with varying transmissivity for hydrogen, we apply the Monte Carlo method in a straightforward fashion: we follow a large number of particles through their life history in the transition region. The particles are represented by their parameters of interest such as speed, direction, position and so on. While the particles proceed through the region, relevant parameters are recorded intermittently. When a sufficient number of particles have been processed, an appropriate census is taken yielding what are essentially histograms representing the distribution of the sample particles in space, velocity, direction and so on. A good general account of the technique was given by Cashwell and Everett (Reference 18) which obviates a detailed account here.

Here we wish only to outline briefly the procedure followed in our Monte Carlo calculation. In accordance with the assumptions made previously, the hydrogen problem is linear (i.e., each hydrogen atom moves independently) interacting only with the particles of the medium. This permits us to follow each atom's life history independently of the others.

We inject hydrogen atoms at the source $(z=0)$ at a random time between 0 and a fixed time $\tau$. The source parameters are drawn randomly from an assumed source parameter distribution. The source parameters are scalar velocity, zenith angle, and "statistical weight". The meaning and significance of the latter will be explained further on. Next, dependent upon our assumptions with respect to the kinetic cross section of hydrogen, we draw a random free path to determine the position of the next encounter with an oxygen atom from a free path distribution appropriate for the medium. If the position so determined is outside the "container" the life history of the particle is terminated without investigating the effects of the projected encounter. If, however, the collision is valid, the parameters of the oxygen atom serving as the hydrogen's partner in the collision are drawn at random consistent with the assumed oxygen equilibrium distribution. The change in the hydrogen atom's parameters is determined from the mechanics of the collision (here, we assume isotropic scattering of the hydrogen in the center of mass system). A new free path is drawn and the procedure is repeated until the $\mathrm{H}$-atom finally leaves the system by either escape at the top or diffusion through the bottom of the container.

The container is divided into layers of width $\Delta z$, each labeled $z_{i}$, the velocity range is divided into intervals $v_{j}$, and intervals are established for the range of zenith angles also. At fixed times (multiples of the fundamental time unit, $\tau$ ) the computation is interrupted and the parameters of the $\mathrm{H}$-atom are recorded. A number of "counter arrays" is set up (each counter is empty at the beginning of the computations, and corresponds to some combination of intervals of space, velocity 
and time previously mentioned), and at recording time $t_{k}$ the statistical weight of the particle is added to the contents of the counter which correspond to the particle's parameters (e.g., if $n\left(v_{i}\right.$, $z_{j}, t_{k}$ ) denotes the weight of particles with velocity in $v_{i}$ in the layer $z_{j}$ at time $t_{k}$, a particle's weight will be added to it, provided that the particle at time, $t_{k}$, possesses the corresponding variables of motion). Because of the assumed linearity of the process, the arrays play a passive role and do not affect the subsequent motion of particles.

The stationarity of the process (i.e., our assumption about the invariability of the oxygen medium) permits a further essential simplification since it enables us not only to superpose the records of different atoms (from linearity), but to superpose records for different times also. This is best explained in the language of stochastic processes.

Let $n_{i}(k)$ be the number of particles with parameters of motion, symbolized by i (at time $\left.t=t_{k}\right)$ be divided by the total number of particles in the system. Then, in the language of Markov processes, it may be termed an "absolute probability" (Reference 19) given by

$$
n_{i}(k)=\sum_{j} n_{j}(0) p_{j}^{(k)}
$$

where $n_{j}(0)$ is the initial distribution, and $p_{j}^{(k)}$ are the stationary, k-step, transition probabilities from state $j$ to state $i$.

Consider now the sum

$$
P_{i}(m)=(m+1)^{-1} \sum_{k=0}^{m} n_{i}(k)=(m+1)^{-1} \sum_{j} \sum_{k} n_{j}(0) p_{j i}^{(k)} .
$$

It is easily shown that $0 \leq P_{i} \leq 1$, and that $\sum_{i} P_{i}=1$, so that $P_{i}$ satisfies the conditions for a probability.

Now let $\mathrm{N}_{j}(\mathrm{~m})$ be the number of particles in state $\mathrm{j}$ at time $\mathrm{m}$. In the framework of our model, this is derived from contributions of the source at times $0,1, \cdots, m-1$. It may then be represented in the form

$$
\begin{aligned}
N_{j}(m) & =\sum_{i_{m}} n_{i}(0)\left(p_{i j}^{(m)} \pm \cdots+p_{i j}^{(2)}+p_{i j}^{(1)}\right) \\
& =\sum_{i} \sum_{n=0}^{m} n_{i}(0) p_{i j}^{(n)} .
\end{aligned}
$$


$\mathrm{N}_{\mathrm{j}}$ (m) is, therefore, of the form of $\mathrm{P}_{\mathrm{j}}$ (apart from a constant), and instead of adding the contributions from the invariable source, characterized by the distribution $n_{i}(0)$, we may sum over the number of particles in state $j$, at various times up to $m$, as is done in Equation 9.

Note that $\mathrm{N}_{\mathrm{i}}(\mathrm{k})$ is a monotonous, non-decreasing function (disregarding fluctuations), but these are of no interest to us at the moment. Now, if we had an infinite number of states and an infinite number of particles, the distribution at $\mathrm{m}$ would yield the required stationary distribution. In practice, we process a finite number of particles, and the lifetime of particles in the container will have a finite maximum, $\mathrm{T}_{\mathrm{m}}$. The actual value of $\mathrm{T}_{\mathrm{m}}$, obtained in the Monte Carlo computation will depend on the distribution of lifetimes and, therefore, will be a function of the number of particles processed, as will be the values for the various $p_{i j}^{(n)}$, which we implicitly apply in the process. Various criteria may serve us to determine the sufficiency of the sample processed, and we shall return to this problem farther on. If we assume that a sufficiently large sample has been processed, we observe from Equation 10 that an approximately stationary state has been reached. After $T_{m}$ the distribution will not change since no further contributions from the initial distribution are obtained.

A further excursion into probability theory will clarify our use of the statistical weight and the assumed source distribution. Under stationary conditions, we might consider escape at the top, not as a final exit from the system but implying transition from there to the source because for each particle escaping another particle has to diffuse into the system. When seen in this light, and in view of the large but finite number of states considered, our model simulates approximately a finite, irreducible, aperiodic Markov chain, which by a theorem of Markov (Reference 20) is ergodic. To test the independence of the stationary distribution of the initial distribution (i.e., the source), we use effectively two different source distributions by applying the following ruse: we draw each particle's parameters from a source flux distribution corresponding to a Maxwellian at the equilibrium temperature, but assign to the particle both a statistical weight 1 , as well as a different statistical weight $w$ which, in effect, alters the source flux distribution in any desired way. As we shall show, the distribution of hydrogen in the transition region is practically independent of the source distribution.

A few words may also be appropriate at this point about both the free path routine used in our computations and the kinetic cross sections utilized in the routine. To achieve a more realistic representation we have taken into account the dependence of cross section on velocity by applying some results obtained in the investigation of transport properties of real gases in recent years by Mason and co-workers (Reference 21). Among others, collision integrals arising in this context have been computed by Krupenie et al (Reference 22) for $\mathrm{O}-\mathrm{H}$ interactions, and these may be readily converted into velocity-dependent cross section (Reference 12). This reduction leads to the approximate form $\sigma=8.1 \times 10^{-16} \times \mathrm{v}^{-0.6} \mathrm{~cm}^{2}$, where $\mathrm{v}$ is measured in kilometers per second. It should be noted that by assuming isotropic scattering in the center of mass frame we have actually represented an atom as a hard sphere, whose diameter is a function of velocity.

We assume that the oxygen in the transition region is in equilibrium at constant temperature. Throughout this region, the extent of which is small in comparison with the earth's radius, gravitational acceleration varies only slightly. One is then justified to assume an oxygen concentration 
varying exponentially with constant scale height, $H$, such that,

$$
N_{0}(z)=-N_{0} e-\frac{z}{H}
$$

where $\mathrm{N}_{0}$ denotes the oxygen concentration at the base of the container, located at $z=0, \lambda(z)$ is the mean free path at $z$ for some particular velocity, $\lambda_{0}$ is the magnitude of the mean free path at $z=0, \theta$ is the zenith angle of a moving particle, and $x=\cos \theta$. If q denotes the survival probability of a particle, that is, the probability of not making a collision over a travel distance $\ell$, the change in q over a pathlength, $d e$, is

$$
\mathrm{dq}=-\frac{\mathrm{q}}{\lambda} \mathrm{d} l=-\frac{\mathrm{q}}{\lambda \mathbf{x}} \mathrm{dz}
$$

Now,

$$
(\lambda)^{-1}=N \sigma=N_{0} \sigma e^{-z / H}=\lambda_{0}^{-1} e^{-z / H} .
$$

Then

$$
\frac{d q}{q}=-\frac{e^{-z / H}}{\lambda_{0} x} d z
$$

which readily integrates to

$$
q(z)=e^{-\left(H / \lambda_{O} x\right)\left(1-e^{-z / H}\right)} .
$$

Obviously, the probability of a collision up to $z$ is given by

$$
\mathrm{P}(\mathrm{z})=1-\mathrm{q}(\mathrm{z})=1-\mathrm{e}^{-\left(\mathrm{H} / \lambda_{\mathrm{O}} \mathrm{x}\right)\left(1-\mathrm{e}^{-z / H}\right)} .
$$

$P(z)$ is a random variable with uniform distribution in $(0,1)$, and the principle of Monte Carlo is readily applied, yielding for the altitude of the first collision of a particle starting at $z=0$

$$
z=-H \ln \left[1-\frac{\lambda_{0} x}{H} \ln s\right] \text {, }
$$

where $s$ is a random number, between 0 and 1 , with the proviso that

$$
\mathrm{s} \geq \mathrm{e}^{-\mathrm{H} / \lambda_{0} x}
$$

If the particle is located at, say, $z=z^{\prime}$, the location of the next collision is given by a formula analogous to Equation 17 with $\lambda_{0}$ replaced by $\lambda_{0} e^{z / H}$, so that

$$
z=z^{\prime}-H \ln \left[1-\frac{\lambda_{o} x}{H} e^{z / H} \ln s\right]
$$


It should be mentioned that in our model calculation we have not taken into account the effect of gravity on the curvature of particle trajectories. This does not mean that gravity has been neglected altogether. The main effect of gravity is the varying concentration of oxygen. In terms of the Boltzmann equation one might say that gravity appears in the collision operator, while the force term $\vec{v} \cdot d f / d \vec{v}$ has been deleted.

\section{RESULTS OF THE MONTE CARLO COMPUTATION}

The Monte Carlo treatment outlined, in contrast to analytical treatments, requires the introduction of certain physical parameters in the course of the calculation itself. Before presenting the results of the computation, we shall describe briefly the atmospheric model used in obtaining our results. The crucial parameters are the temperature of the medium (predominantly oxygen) and the number density of ambient oxygen. The large amount of information gained in recent years permits a high degree of confidence in the agreement of numerical results with the actual physical conditions prevailing in the upper atmosphere.

For computation in this study, two typical cases were chosen: an isothermal atmosphere at $1500^{\circ} \mathrm{K}$, and one at $2500^{\circ} \mathrm{K}$. This choice was motivated by the desire to gain insight into the escape problem both under average conditions, and under rather extreme conditions at solar maximum represented by the higher temperature. The atmospheric data for the $1500^{\circ}$ case are based on a variety of sources (References 14, 23, and 24) which show satisfactory numerical agreement between the various authors.

Data for the high temperature case were deduced by extrapolation from the cited study of Harris and Priester (Reference 24) of the time-dependent atmosphere. The relevant parameters, $N_{0}, \lambda_{0}$, and $\mathrm{H}$ are represented in Table 1.

As mentioned previously, the choice of proper boundaries for the computation is intimately connected with the consistency of the model. The effect of escape on the distribution should not be critically dependent on the particular choice of its altitude because, as we know, the sharp escape level is a convenient simplification and should not be taken too literally. In practice, this will be a rather extended diffuse region. If we are able to demonstrate that departure from approximate diffusive equilibrium (ascribed to the effect of escape) occurs independently of the exact boundary, our confidence in the meaningfulness of our results will be greatly strengthened. Figures 1 and 2 show the variation of the hydrogen concentration for both temperatures and various upper boundaries. The statistics of these computations are not too accurate, but apart from curve 1 in Figure 1, they show good 
agreement in the upper part of the transition region. They also display a significant change in slope of the concentration curve to occur in a definite region, the location of which is independent of the boundaries chosen. This leads to the conclusion that the increasing dilution of the ambient oxygen results in a critical region where escape affects significantly the distribution of hydrogen. Application of the usual criteria for the choice of the base of the exosphere (References 11 and 12) reveals that this region is located close to, but below this level. This is consistent with our observation that escape will result in a changed distribution at the exospheric base, which has to be taken into account before applying methods appropriate to the collisionless domain. In the low temperature case, this break is quite sharp with the critical region located in the vicinity of 500 $\mathrm{km}$, while the exospheric base, in agreement with our atmospheric model, it is around $580 \mathrm{~km}$. The transition in the $2500^{\circ}$ case is less pronounced, occurring around $700 \mathrm{~km}$; its relative smoothness appears to be a consequence of the considerably increased scale height of oxygen. As Figure 2 reveals, the choice of the lower boundary has some import on the results in the lower part of the region of interest (but does not affect the upper portion). If we wish to join our results to a curve obtained from approximate diffusive equilibrium, this choice has to be done with sufficient care. We cannot go into the detailed considerations of this problem here, but the choice of the particular level adopted has little effect on the velocity distribution since the concentration of the

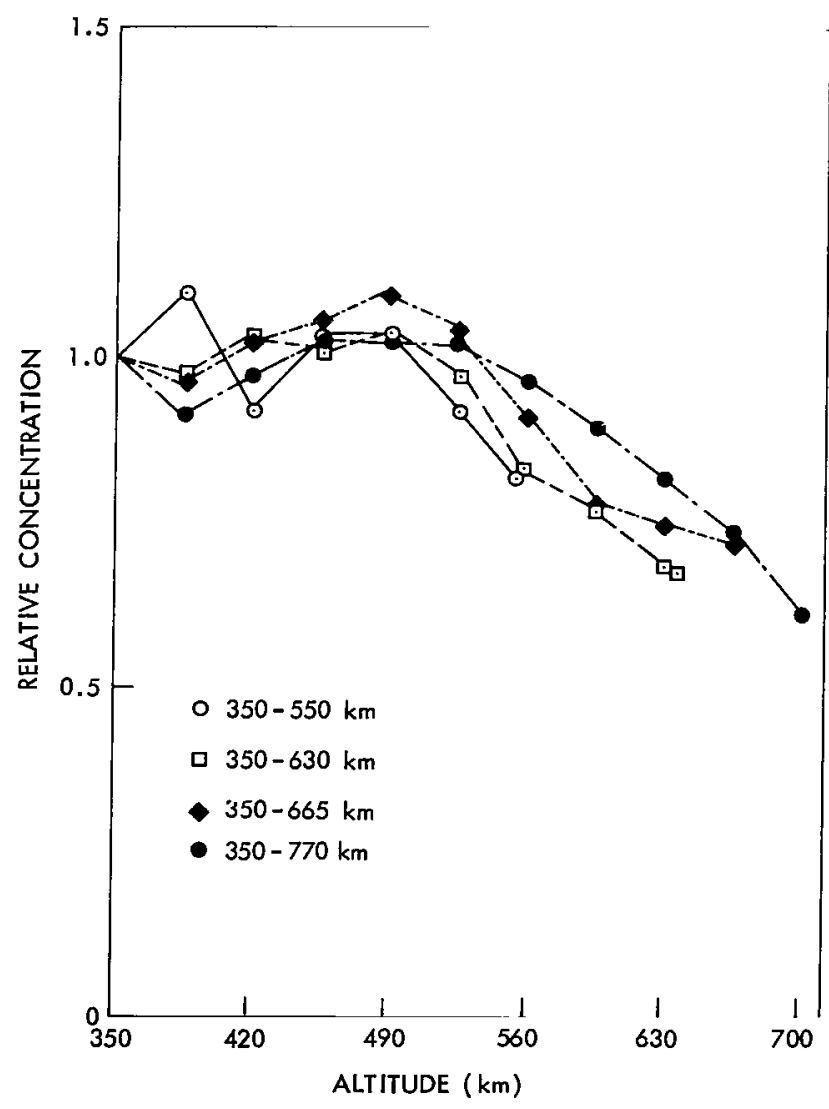

Figure 1-Relative hydrogen concentration versus altitude for different boundaries. predominant constituent in the lower region is high enough to ensure effective randomization of hydrogen velocities.

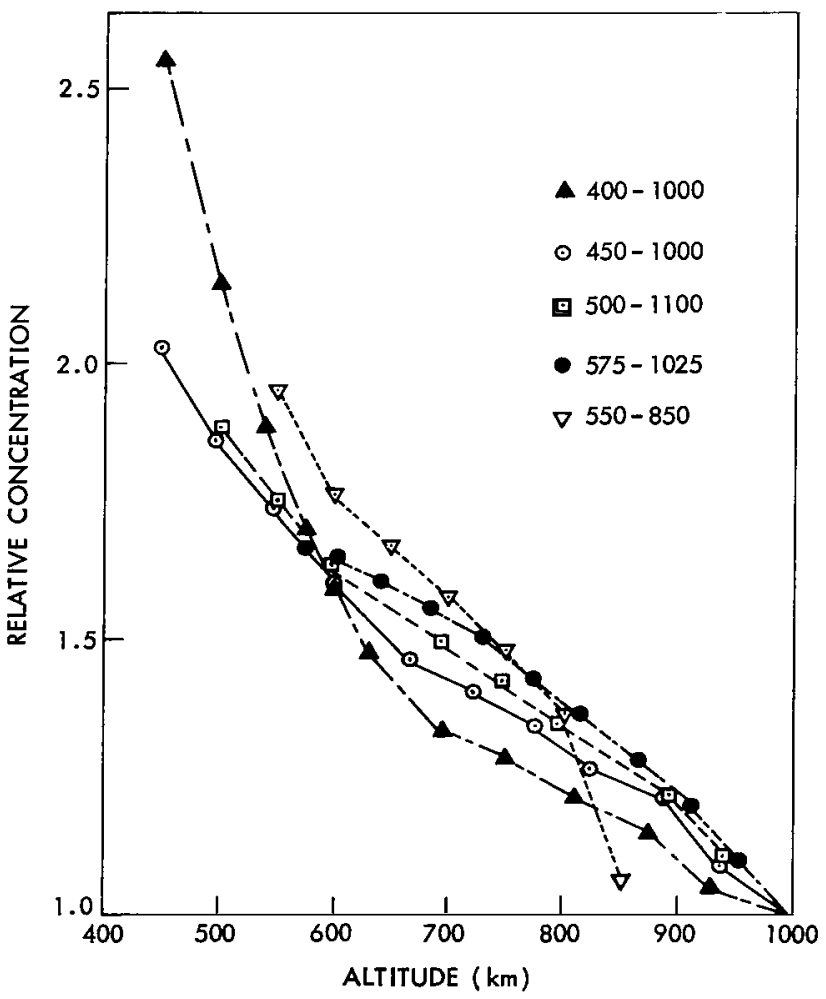

Figure 2-Relative hydrogen concentration versus altitude for different boundaries. 
In accordance with the above considerations, the levels chosen as boundaries in our computation were $350 \mathrm{~km}$ and $700 \mathrm{~km}$ for the $1500^{\circ}$ case, and $575 \mathrm{~km}$ and $1025 \mathrm{~km}$ for the 2500 case.

Of great significance, of course, is the problem of sample size required for processing in a computation of this character and the statistical accuracy achieved. As we observed earlier, our approximation to a realistic picture of a stationary state will depend quite strongly on the size of the sample. Continuous, endless replication of the Monte Carlo procedure should yield exact results within the framework of the underlying physical assumptions, but the approach to limiting accuracy becomes gradually slower and slower. It is necessary, therefore, to choose some suitable criteria for termination of the computations. Since the problem at hand is of greater complexity than relatively simple cases amenable to well defined mthods such as sequential analysis (Reference 25), we were forced to be guided by some intuitively plausible criteria although we were unable to put them into rigorous mathematical terms. It has to be borne in mind, moreover, that for given sample size, the accuracy achieved will vary with the nature of the statistic investigated. The sample size required for a realistic estimate of the relative concentration, for instance, will be much smaller than that necessary for a good estimate of the angular distribution of particles in a particular velocity range and a particular layer. Since we were interested in a great range of statistics, our sample size is on the order of hundreds of thousands of source particles, the bulk of which does not penetrate too far into the medium, and is swiftly scattered back through its lower boundary.

An idea of the stability and accuracy obtained in the computations can be gained from inspection of Tables 2 and 3, which represent the relative concentration in selected layers of the medium versus the number of source particles processed. It appears that for this particular statistic we are within an accuracy of $1 \%$.

Table 2

Variation of the Relative Hydrogen Concentration in Selected Layers with the Number of Source Particles in the $1500^{\circ} \mathrm{Case}$.

\begin{tabular}{|c|r|r|r|r|r|}
\multirow{2}{*}{$\begin{array}{c}\text { Number of Source } \\
\text { Particles }\end{array}$} & \multicolumn{5}{|c|}{ Layer (kilometers) } \\
& $378-385$ & $448-455$ & $518-525$ & $588-595$ & $658-665$ \\
20036 & & & & - \\
46173 & .939347 & 1.035000 & .998599 & .800252 & .700939 \\
64173 & .979458 & .990204 & .996831 & .796101 & .694602 \\
90000 & .969872 & .984525 & .994282 & .802329 & .695022 \\
120000 & .970037 & .989772 & .999978 & .807954 & .697872 \\
150000 & .976554 & 1.000267 & 1.011560 & .814299 & .706973 \\
180000 & .994485 & 1.015202 & 1.004433 & .837367 & .723087 \\
210000 & 1.000058 & 1.014311 & .997391 & .843658 & .726010 \\
240000 & 1.012421 & 1.012463 & .994761 & .843256 & .724136 \\
270000 & 1.010880 & 1.010340 & .986317 & .846516 & .717696 \\
300000 & 1.006388 & 1.000687 & .968360 & .834693 & .703233 \\
330000 & 1.015932 & 1.008722 & .976175 & .844235 & .715809 \\
360000 & 1.010353 & 1.004137 & .970938 & .841901 & .714477 \\
390000 & 1.012284 & 1.007843 & .972897 & .847781 & .719022 \\
& 1.016374 & 1.010825 & .970580 & .848068 & .716457 \\
\hline
\end{tabular}


Table 3

Variation of the Relative Hydrogen Concentration in Selected Layers with the Number of Source Particles in the $2500^{\circ}$ Case.

\begin{tabular}{|c|c|c|c|c|c|}
\hline \multirow{2}{*}{$\begin{array}{c}\text { Number of Source } \\
\text { Particles }\end{array}$} & \multicolumn{7}{|c|}{ Layer (kilometers) } \\
\cline { 2 - 6 } & $611-620$ & $701-710$ & $791-800$ & $881-890$ & $971-980$ \\
\hline 21500 & & & & \\
67000 & 1.043191 & .917147 & .860685 & .744332 & .650085 \\
100000 & .963635 & .897185 & .842384 & .747455 & .616731 \\
130000 & .973088 & .904620 & .858624 & .751596 & .629521 \\
160000 & .979538 & .913705 & .855260 & .754563 & .632597 \\
190000 & .979349 & .915424 & .857541 & .754975 & .632721 \\
220000 & .976984 & .913239 & .856988 & .753267 & .634049 \\
250000 & .975521 & .910686 & .853568 & .751488 & .633437 \\
280000 & .974909 & .911627 & .847652 & .746549 & .629097 \\
310000 & .975148 & .907655 & .845661 & .743627 & .625194 \\
350000 & .979932 & .911077 & .847217 & .743735 & .626639 \\
& .973840 & .906308 & .842933 & .741363 & .623740 \\
\hline
\end{tabular}

The results of the computation are best classified roughly into two categories: microscopic results and macroscopic results. By the former we mean, primarily, the detailed velocity distribution as a function of altitude, i.e., quantities which are not likely to be amenable to direct measurement in the near future.

We present first, therefore, the salient features of the macroscopic results such as the variation of hydrogen concentration with altitude and typical relaxation times as well as diffusion times and velocities.

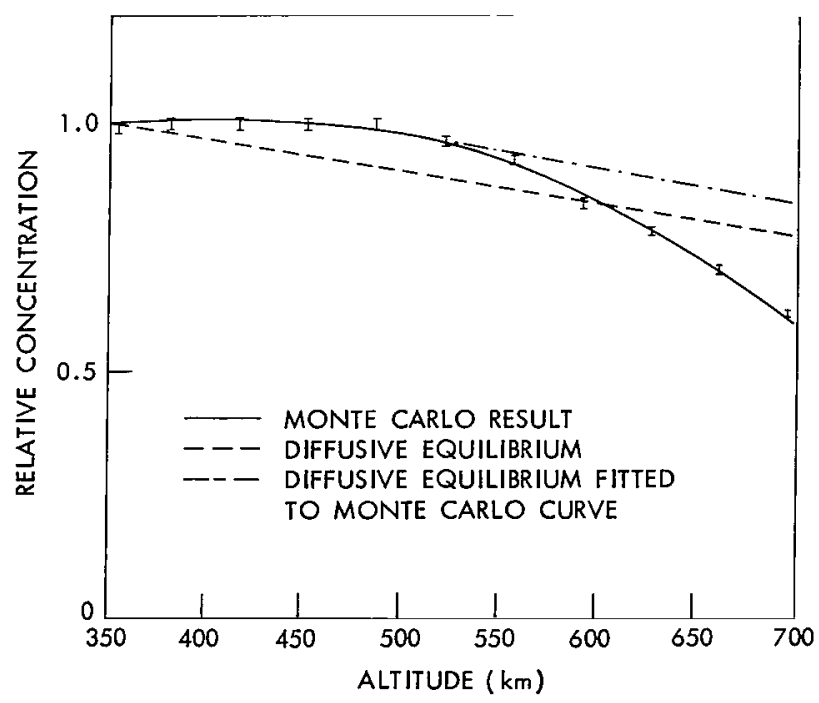

Figure 3-Relative hydrogen concentration versus altitude for $1500^{\circ} \mathrm{K}$.
Figures 3 and 4 present the relative concentration of hydrogen versus altitude in the transition region for the two cases treated. As can be seen, the departure from diffusive equilibrium is not too pronounced. The significance of the departure lies, however, in the increased rate of change as one ascends in the transition layer. Previous work (References 14 and 26) implicitly assumed that with increasing altitude and growing distance of the physical source of hydrogen at about $100 \mathrm{~km}$, the approach to diffusive equilibrium becomes closer and closer. Our results indicate that this is not the case; equilibrium cannot be attained when collisions become increasingly rare, and the effects of selective removal make themselves felt. As Figure 4 
shows the smooth fit to an equilibrium curve at the lower end has been achieved here with a high degree of success. In the other case we were not quite aware of the implications of this problem when calculations were started, and in this case our results should be fitted to the diffusion curve at a level between 450 and $500 \mathrm{~km}$. As remarked above, this does not significantly affect the results from the critical region around $500 \mathrm{~km}$ on.

Figure 5 presents a typical curve of how the concentration in a layer varies with time. It is seen that it is very close to a time dependence of the form $1-e^{-t / \tau}$. If, on this basis, we

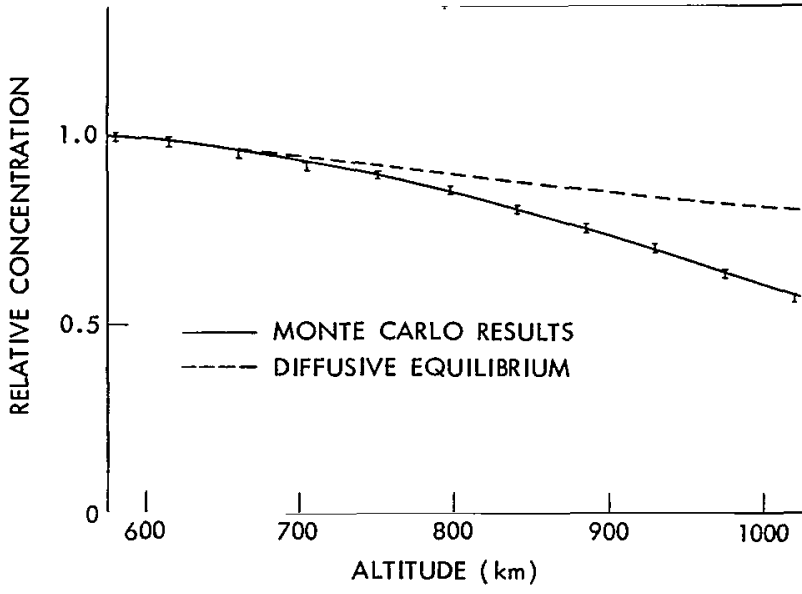

Figure 4-Relative hydrogen concentration versus altitude for $2500^{\circ} \mathrm{K}$. compute the numerical values of the relaxation times, we obtain from a least square calculation for selected layers the numerical values presented in Tables 4 and 5. As is to be expected, the lower layers, where collisions are frequent, approach stationary conditions faster than the upper, rarer layers. If we denote by $n(t)$ the number content of the transition region at time $t$, and by $n_{f}$ the content upon reaching a steady state, a plot of $1-\left[n(t) / n_{f}\right]$ is of the form presented in Figure 6. As we observe from these curves, the times for attainment of a high degree of stationarity, say $90 \%$, vary from $\sim 2500$ seconds at $2500^{\circ}$ to $\sim 1500$ seconds at $2500^{\circ}$. The short times involved indicate that the distribution of the minor constituent follows very closely the thermal conditions prevailing in the main atmosphere, which should be taken into account when attempting to calculate the lateral flow of hydrogen due to concentration gradients (References 27 and 28). In reality, due to the time variations of the main atmosphere (which seem to be well understood (References 24 and 29)) stationary conditions will never be

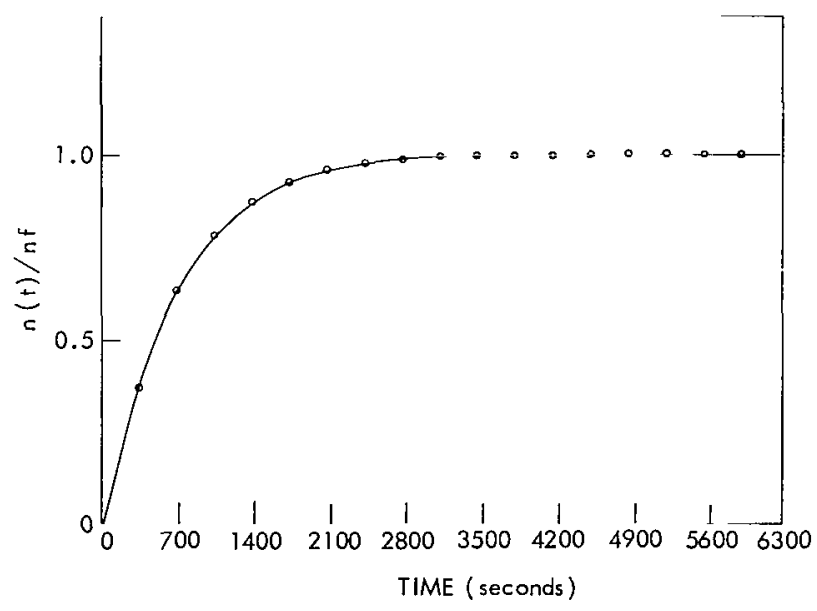

Figure 5-Typical curve showing the hydrogen concentration-versus-time approach to steady state.

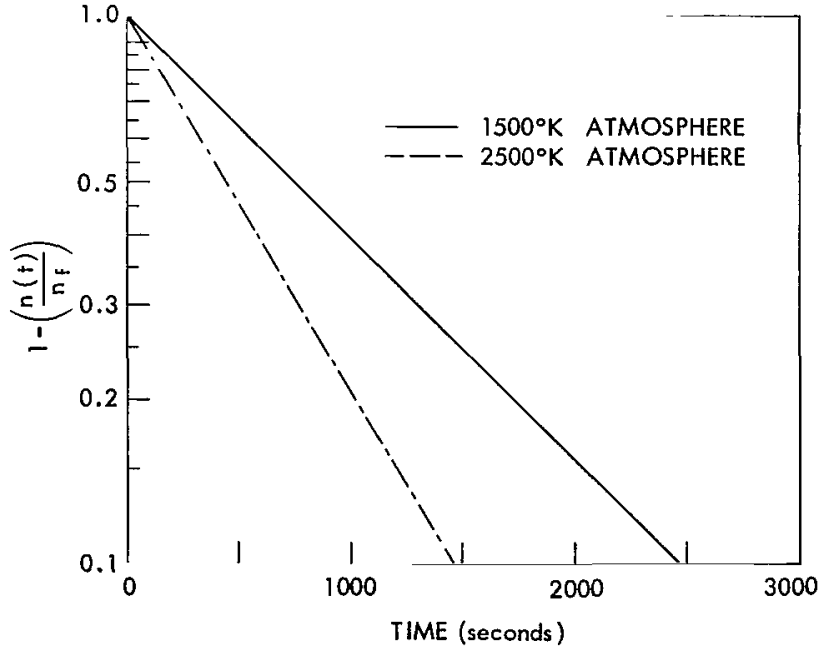

Figure 6-Transition region-versus-time approach to steady state. 
Table 4

Relaxation Times of Selected Layers in the $1500^{\circ}$ Case.

\begin{tabular}{|c|c|c|c|c|c|}
\hline Layer (km) & $378-385$ & $448-455$ & $518-525$ & $588-595$ & $658-665$ \\
\hline$t(\mathrm{sec})$ & 943.0 & 1096.0 & 1150.0 & 1222.4 & 1192.1
\end{tabular}

Table 5

Relaxation Times of Selected Layers in the $2500^{\circ}$ Case.

\begin{tabular}{|c|c|c|c|c|c|}
\hline Layer (km) & $611-620$ & $701-710$ & $791-800$ & $881-890$ & $971-980$ \\
\hdashline-- & & & & & \\
\cline { 1 - 2 }$(\mathrm{sec})$ & 570.6 & 637.6 & 660.1 & 666.2 & 659.2 \\
\hline
\end{tabular}

fully attained, but the approach to them appears faster than was thought hitherto, which would prevent the smoothing out of local differences due to lateral flow. A final answer to this problem can be obtained only after extension of this study to the time dependent case.

In regard to the problem of diffusion times, in our computation we have obtained the mean time of travel of escaping particles between the lower and upper boundaries of the transition region. These correspond to mean diffusion velocities as follows:

$$
\begin{aligned}
& \sim 3.4 \times 10^{4} \mathrm{~cm} \mathrm{sec}^{-1} \text { for } 1500^{\circ} \text { case } \\
& \sim 9.0 \times 10^{4} \mathrm{~cm} \mathrm{sec}^{-1} \text { for } 2500^{\circ} \text { case. }
\end{aligned}
$$

Note should be taken that these mean diffusion velocities are not identical with the mean flow velocity of hydrogen, except, ideally at the escape level itself where escaping particles provide all the net flow. The former represents an average taken only over those particles which ultimately pass through the upper boundary, and does not take into account those hydrogen atoms which are turned back at one or another location in the "container" thus terminating their life history by passing downward through the lower boundary.

We now turn to a review of the main microscopic results. In our computations we obtained the distributions of various parameters in selected layers spaced about equally throughout the region of interest. The width of these layers was chosen such that their kinetic depth varied from about a mean free path near the lower boundary to a small fraction of a mean free path near the upper boundary; this is in accordance with the assumption that changes in the distribution will be faster near the escape level. The parameters whose distribution was obtained, were the following: ver tical velocity, horizontal velocity, total velocity, and angular distribution of various velocity groups. For the distribution of the vertical velocity component, the double bookkeeping procedure outlined previously was adopted.

Samples of the distributions are presented in Figures 7 through 12. 

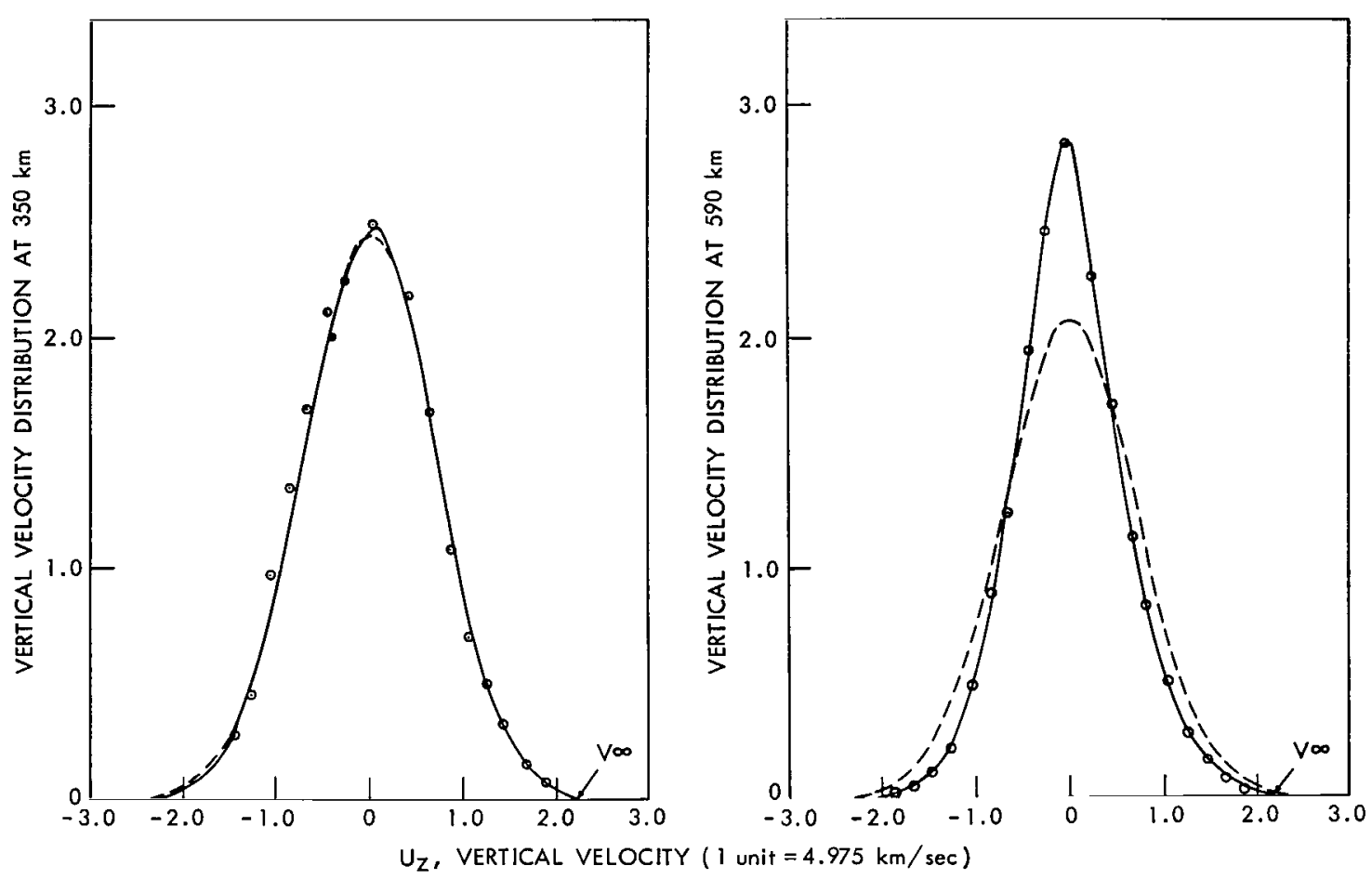

Figure 7-Distribution of vertical velocity for $1500^{\circ} \mathrm{K}$ case (dashed curve - Maxwellian at $1500^{\circ} \mathrm{K}$ ).

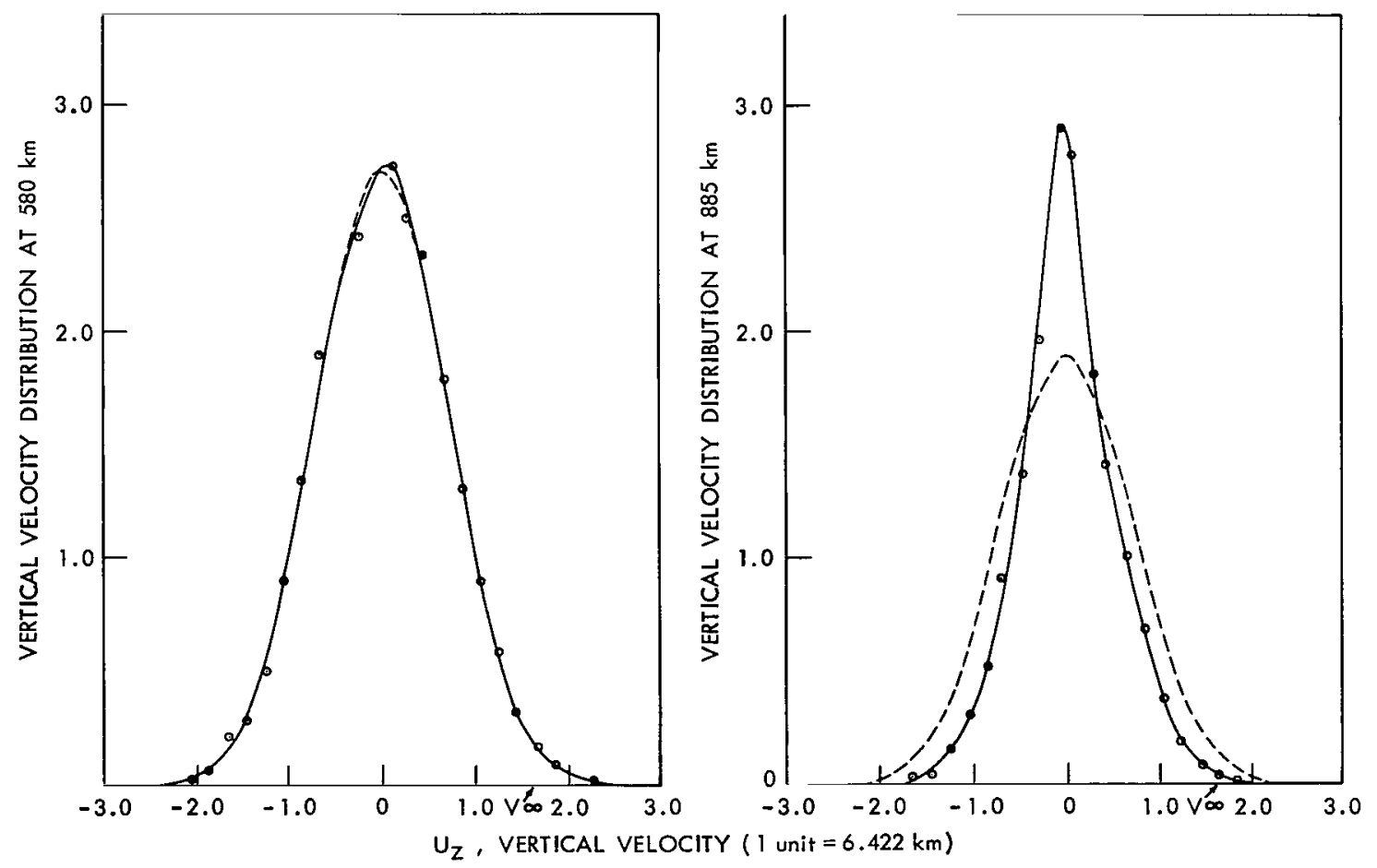

Figure 8-Distribution of vertical velocity for $2500^{\circ} \mathrm{K}$ case (dashed curve - Maxwellian at $2500^{\circ} \mathrm{K}$ ). 


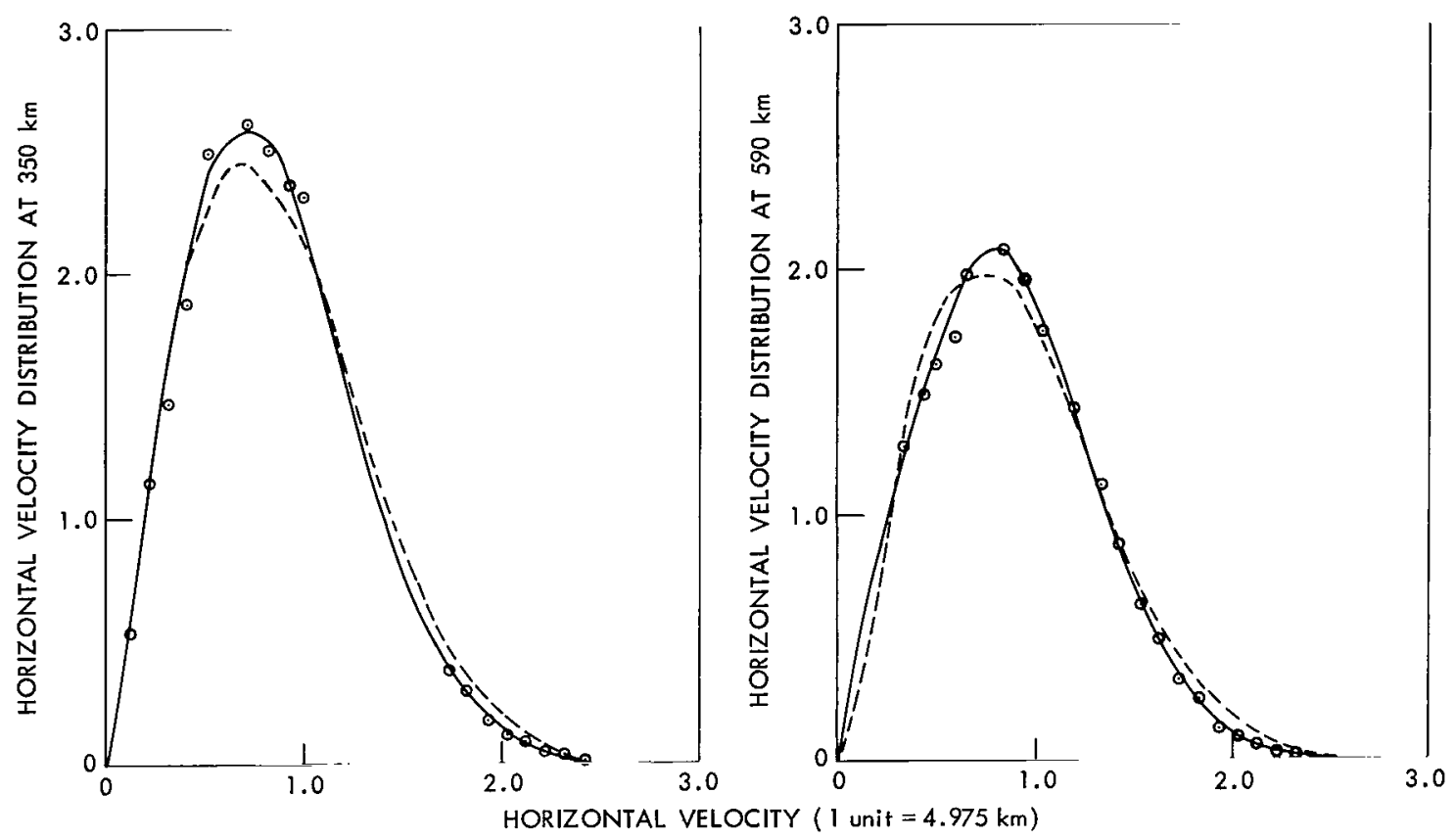

Figure 9-Distribution of horizontal velocity for $1500^{\circ} \mathrm{K}$ case (dashed curve - Maxwellian at $1500^{\circ} \mathrm{K}$ ).

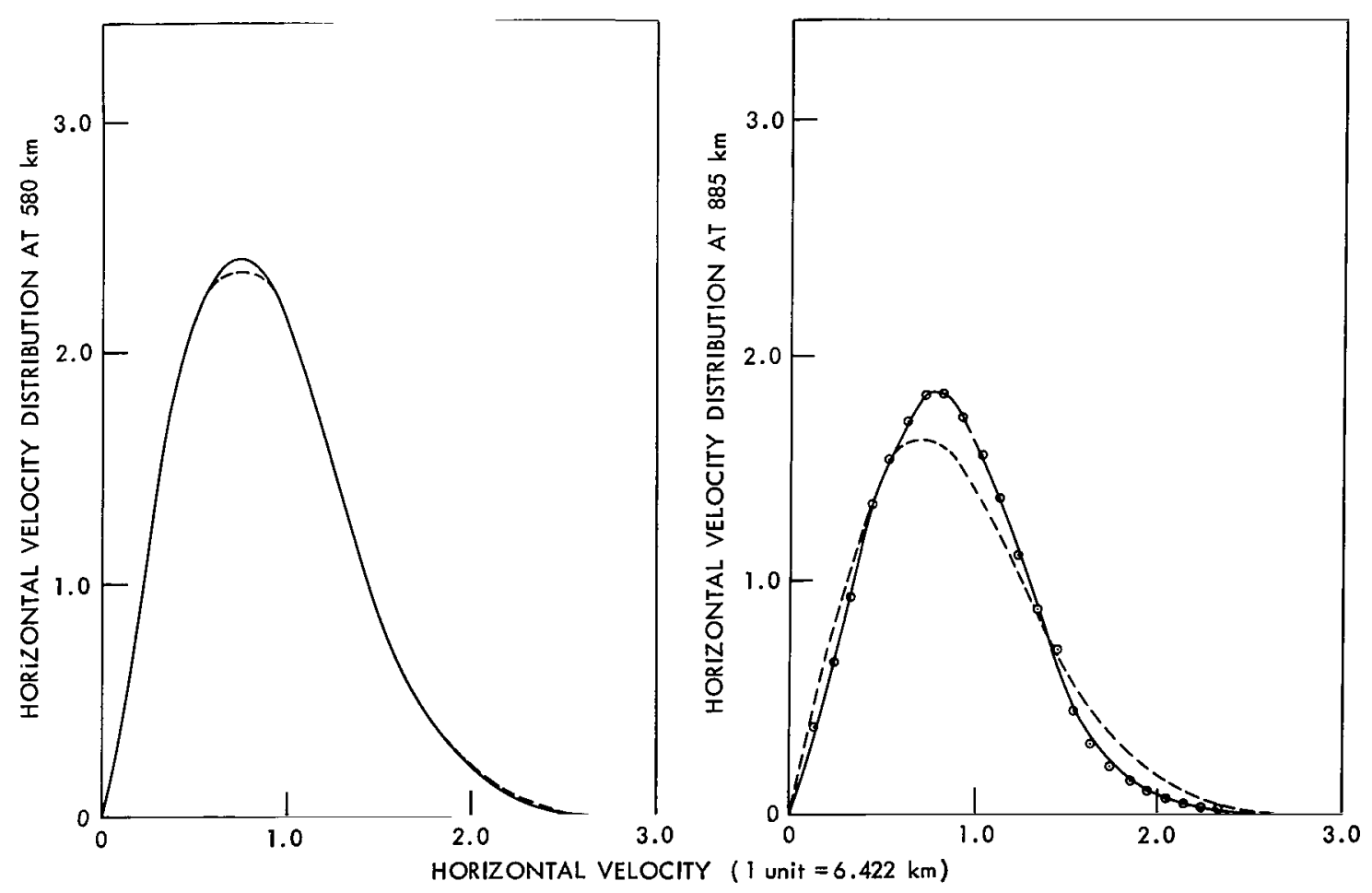

Figure 10-Distribution of horizontal velocity for $2500^{\circ} \mathrm{K}$ case (dashed curve - Maxwellian at $2500^{\circ} \mathrm{K}$ ). 


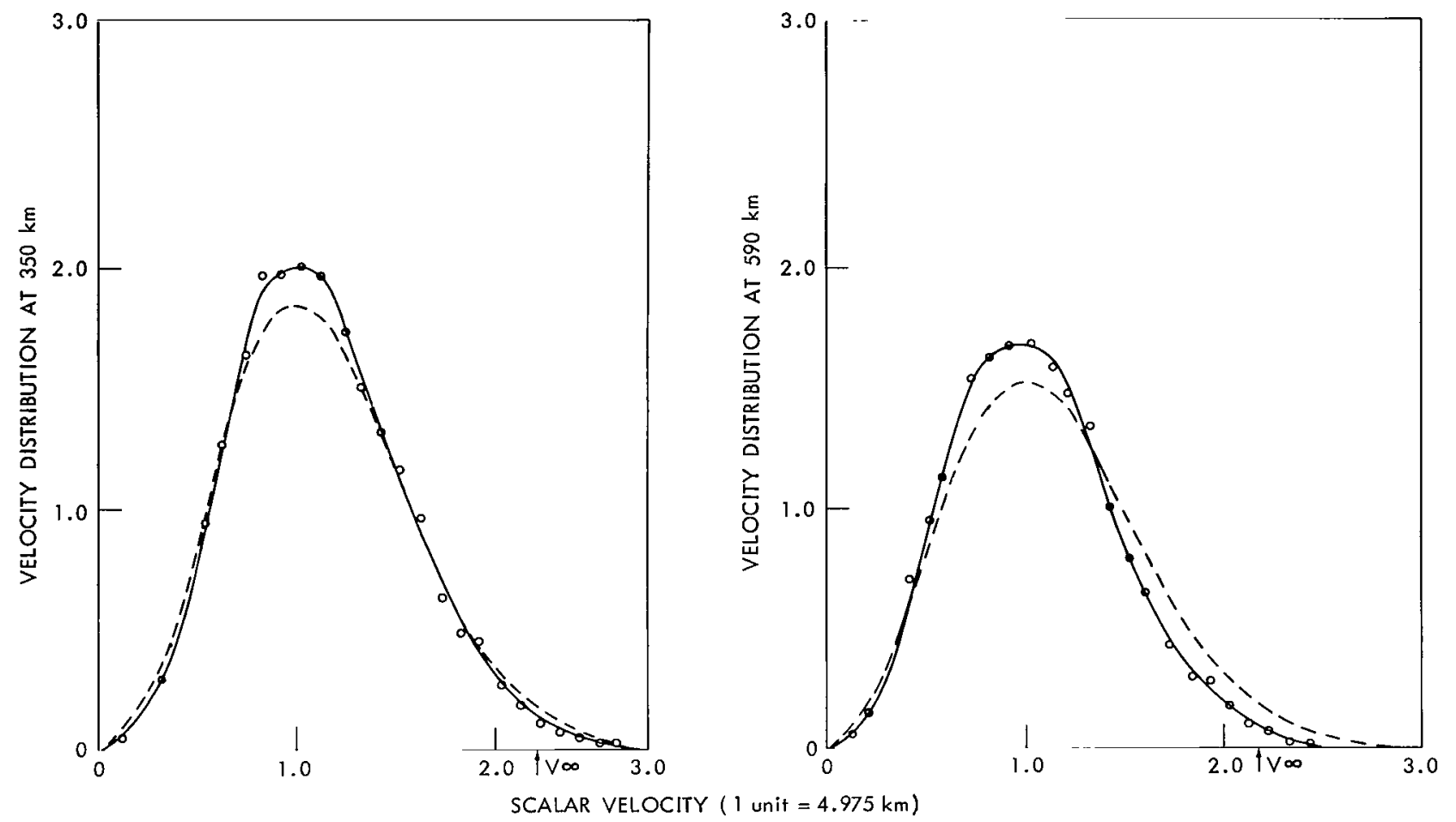

Figure 11-Distribution of scalar velocity for $1500^{\circ} \mathrm{K}$ case (dashed curve - Maxwellian at $1500^{\circ} \mathrm{K}$ ).

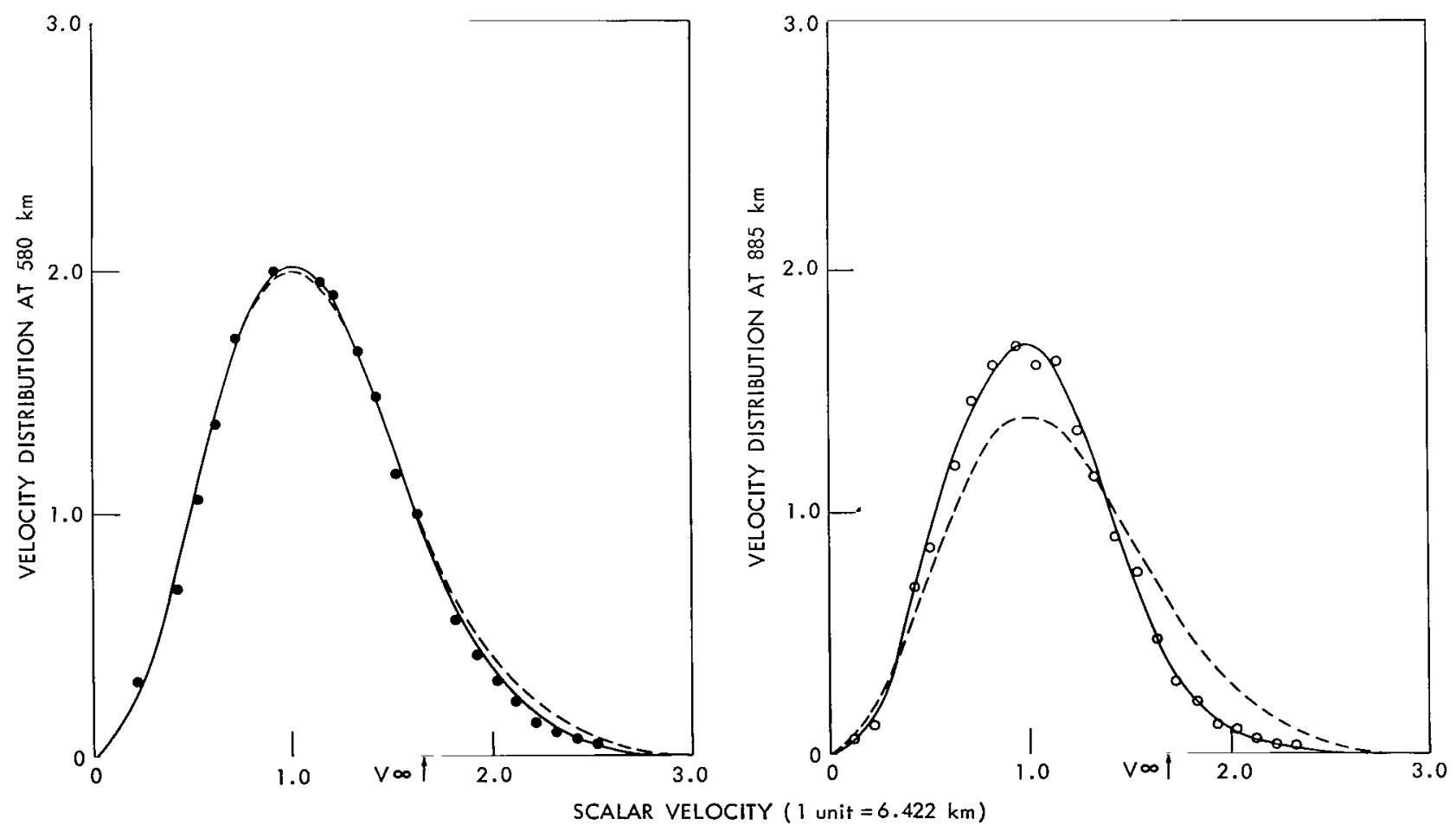

Figure 12-Distribution of scalar velocity for $2500^{\circ} \mathrm{K}$ case (dashed curve - Maxwellian at $2500^{\circ} \mathrm{K}$ ). 
These figures are arranged in pairs; one member of the pair referring to the distribution at a level close to the lower boundary, the other referring to a level in the vicinity of the assumed level of the exospheric base. In all these figures a comparison curve is included, showing a distribution corresponding to equilibrium at the temperature of the ambient oxygen, and normalized to the area under the curve obtained in our computations. The unit of velocity used in these curves is $(2 \mathrm{kT} / \mathrm{m})^{1 / 2}$ (i.e., $4.975 \mathrm{~km} / \mathrm{sec}$ at $1500^{\circ} \mathrm{K}$ and $6.422 \mathrm{~km} / \mathrm{sec}$ at $2500^{\circ} \mathrm{K}$ ). Vertical units are arbitrary, but are the same for both members of a pair so that the ratio of their areas corresponds to the ratio of hydrogen concentrations at the corresponding levels.

Figure 7 shows the computed distribution of the vertical velocity component, $\mathrm{v}_{z}$, around $350 \mathrm{~km}$ and $590 \mathrm{~km}$ for the $1500^{\circ}$ case. At the lower level the fit to the assumed Maxwellian is almost perfect. In the computed curve, there is only a slight asymmetry which is needed to sustain a net flow. At the higher level where both the departure from equilibrium and the asymmetry become quite pronounced, the disagreement between the curves mount with increasing velocity. Even more striking is this effect in the high temperature case presented in Figure 8, where the depletion of the high velocity tail of the distribution can be better observed.

Figures 9 and 10 display the behavior of the horizontal velocity for both cases treated. Evidently, in view of the dependence of escape on direction, the distribution of this component is far less altitude dependent than that of the vertical component. Corresponding figures for the distribution of total velocity are Figures 11 and 12, which reveal a degree of departure from equilibrium intermediate between those of the separate distributions.

In the absence of an equilibrium distribution, isotropy is destroyed and the angular distribution becomes a function of velocity. Insight into this effect can be gained from Figure 13 which presents the angular distribution of narrow velocity groups centered around $\mathrm{v}=1.1$ and $\mathrm{v}=2.1$ (in the units described above) for the high temperature case. The lower velocity is near the mean velocity, while the higher velocity is beyond escape velocity. The marked change in the angular distribution is characteristic of the process studied: in the lower region, for low and moderate velocities, the angular distribution is isotropic for all practical purposes; in the higher velocity range a slight degree of anisotropy may be observed. In the escape region, anisotropy is destroyed for all velocities, yet, in the velocity range below escape velocity, symmetry in the forward and backward directions is conserved while beyond this limit asymmetry is most pronounced; this is in agreement with the assumptions made earlier.

To obtain a more quantitative estimate of the departure from equilibrium the results were subjected to thorough statistical analysis, which confirmed the trend in behavior of the escaping gas. An example of this is presented in Table 6. In this table we present the results of a $x^{2}$ test, as well as a Kolmogorov-Smirnov test for the hypothesis that our computed distribution is a sample of an equilibrium distribution at the temperature of the oxygen medium. The numbers appearing in the table are, in effect, the probabilities that this hypothesis is true in selected layers of the transition region. 

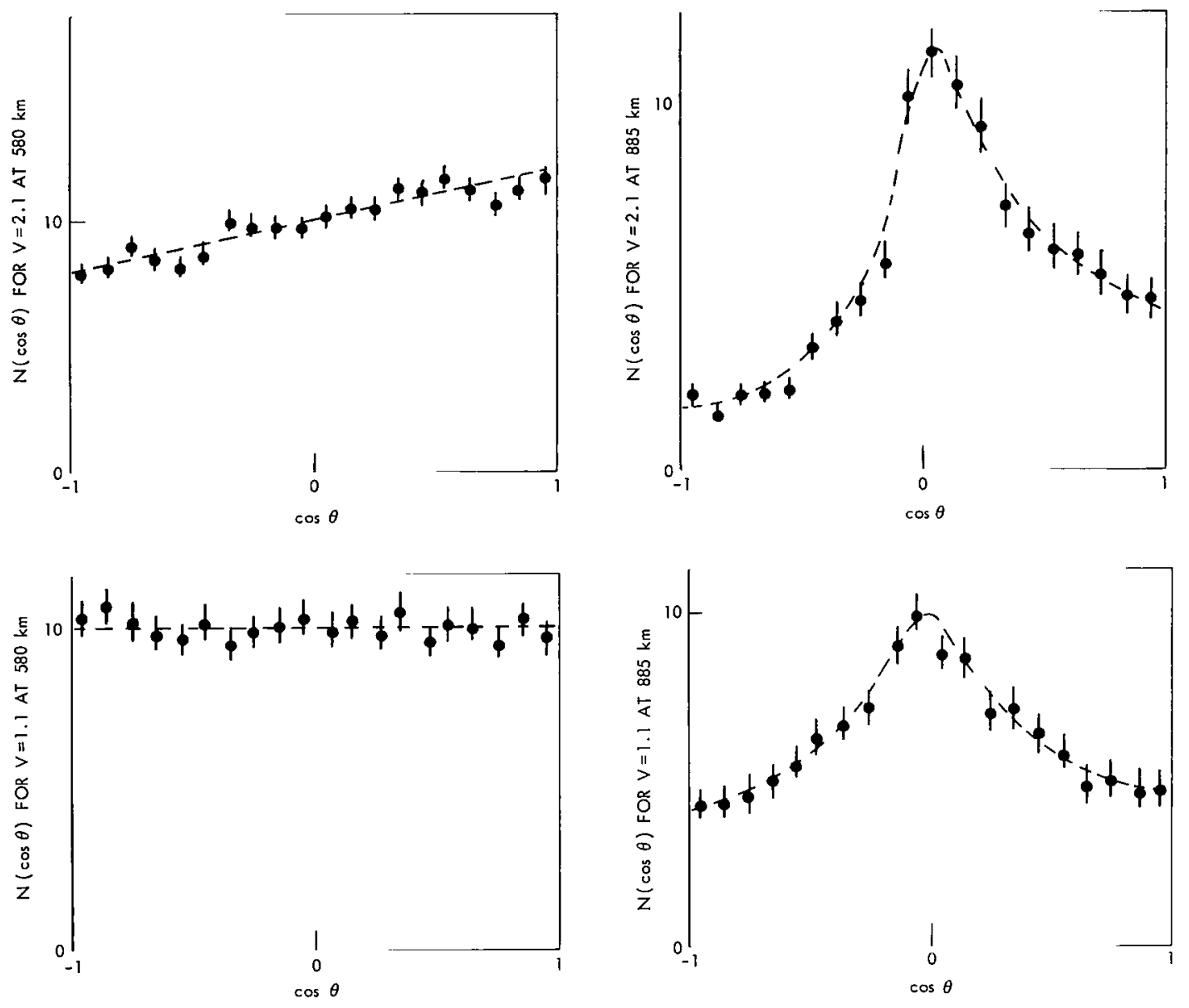

Figure 13-Typical Angular Distribution Curves.

Table 6

Significance Levels for the Distribution of Vertical and Horizontal Velocity at $1500^{\circ} \mathrm{K}$.

\begin{tabular}{l|} 
\\
Layer $(\mathrm{km})$ \\
\\
\\
$350-357$ \\
$378-385$ \\
$413-420$ \\
$448-455$ \\
$483-490$ \\
$518-525$ \\
$553-560$ \\
$588-595$ \\
$623-630$ \\
$658-655$ \\
$693-700$
\end{tabular}

\begin{tabular}{l|l}
\multicolumn{2}{|c}{ Vertical Velocity } \\
\multicolumn{1}{|c|}{$x^{2}$} & \multicolumn{1}{|c}{$\mathrm{K}-\mathrm{S}$} \\
& \\
0.720 & 0.500 \\
0.310 & 0.027 \\
0.360 & 0.250 \\
0.038 & 0.025 \\
0.002 & 0.001 \\
0.001 & $<0.001$ \\
$<0.001$ & $<0.001$ \\
$<0.001$ & $<0.001$ \\
$\ll 0.001$ & $\ll 0.001$ \\
$\ll 0.001$ & $\ll 0.001$ \\
$\ll 0.001$ & $<0.001$
\end{tabular}

Horizontal Velocity

\begin{tabular}{|c|l|}
\hline$x^{2}$ & $\mathrm{~K}-\mathrm{S}$ \\
& \\
0.090 & 0.085 \\
0.035 & 0.029 \\
0.042 & 0.051 \\
0.013 & 0.021 \\
0.032 & 0.027 \\
0.024 & 0.030 \\
0.009 & 0.007 \\
0.011 & 0.008 \\
0.010 & 0.010 \\
0.007 & 0.005 \\
0.006 & 0.003 \\
\hline
\end{tabular}


Of great value in our interpretation of the escape process, are further results of the statistical analysis. Since temperature is, in essence, a measure of the dispersion of velocities, we may go one step further and define an effective temperature for the separate directions of velocity as measured in terms of the dispersion of these velocity components. The results of this approach are presented in Figures 14 and 15. It may be observed from these figures that the vertical ef-

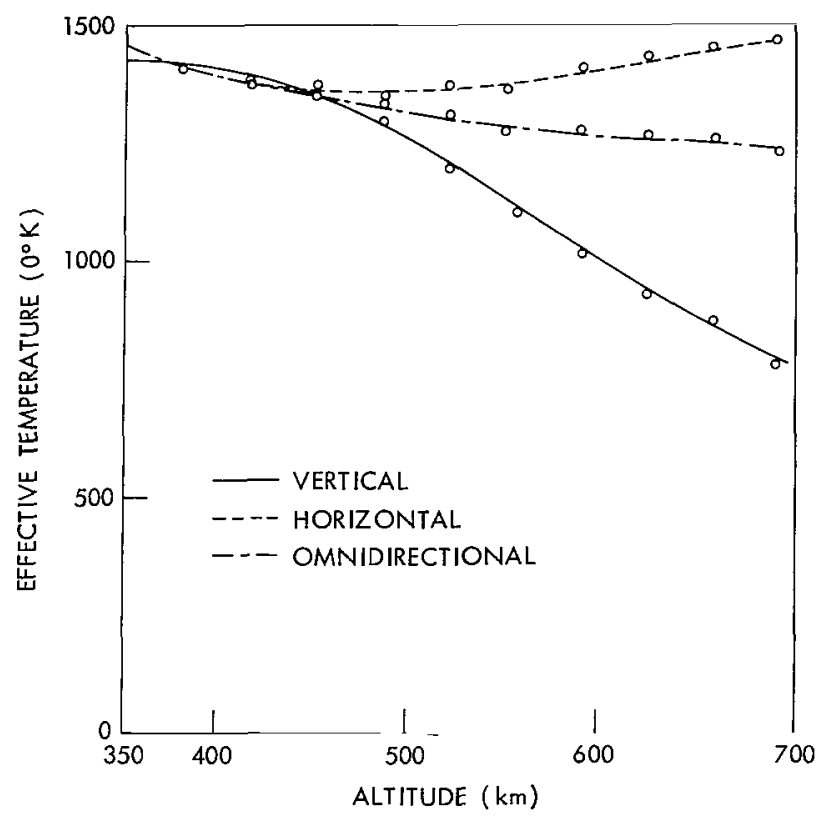

Figure 14-Effective temperatures versus altitude for $1500^{\circ} \mathrm{K}$ case.

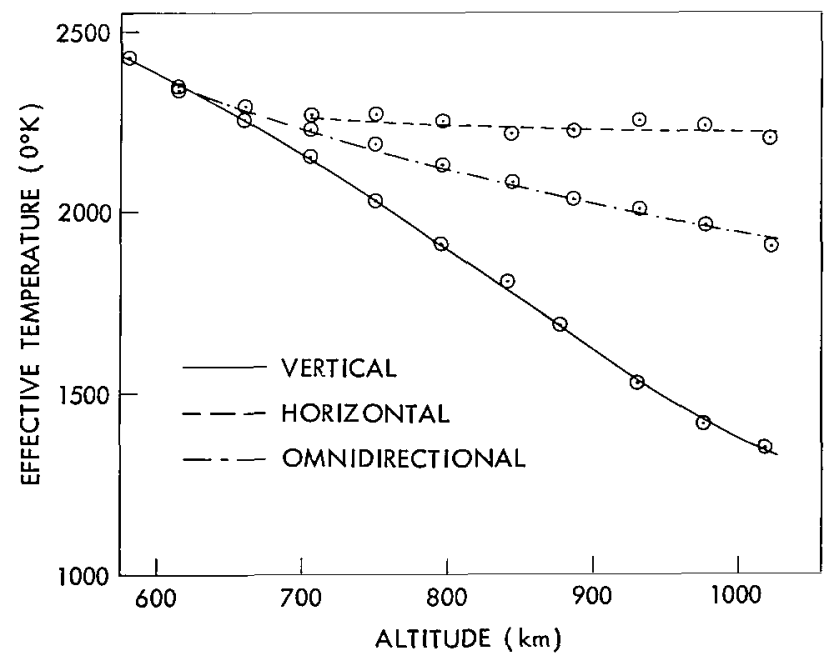

Figure 15-Effective temperatures versus altitude for $2500^{\circ} \mathrm{K}$ case. fective temperature decreases steadily, whereas, the horizontal effective temperature decreases initially and remains more or less constant thereafter. This is what we term anisotropic cooling of the escaping gas; in other words, we might say that temperature is a function of direction and that the rate of change of temper ature varies with direction also. In any further investigation of the escape problem this aspect may well serve as a convenient starting point.

As a final sample of our results, we present Figure 16, a graph of the normalized net flux in the transition region for the case of $2500^{\circ}$. As required by steady state conditions, this is constant within the limits of our statistical accuracy; it is another indication of the consistency of our results. No such curve is presented for the $1500^{\circ}$ case, because in view of its small magnitude, the statistical accuracy in our determination of the mean flow velocity is much poorer.

We close with a remark pertaining to the loss rate of hydrogen. Table 7 presents the effusion velocities of hydrogen at the base of

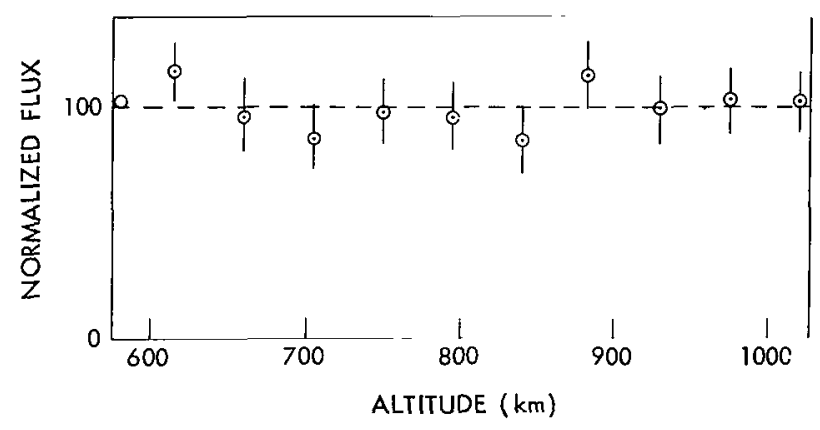

Figure 16-Normalized net hydrogen flux versus altitude for $2500^{\circ} \mathrm{K}$ case. 
the exosphere based on the calculations of Kockarts and Nicolet (Reference 14), as well as the results of the present calculations.

It may be somewhat surprising that the (relative) discrepancy is greater in the low temperature case, but one should bear in mind that in this case the quantities involved are so small that a small absolute change may produce a great relative change. Moreover, the escape process is, to a certain extent, self-governing. The changes in the distribution resulting from escape reduce escape. Within the limits of our model's validity, we observe that the loss rates based on equilibrium appear to be an overestimate by a factor of 3 or more in the range of temperatures covered by this study.

\section{ACKNOWLEDGMENTS}

We wish to express our thanks to the U. S. Weather Bureau, the National Aeronautics and Space Administration and the Computer Science Center at the University of Maryland for their financial support which made this project possible. We are greatly indebted to $\mathrm{Mr}$. Lee N. Foster for his invaluable help in computer programming and otherwise, and to Dr. W. N. Hess for his continued interest in the problem. We also appreciate many helpful suggestions of Dr. E. A. Mason, Dr. S. Weissman, Dr. D. A. Tidman and Dr. A. W. DeSilva.

(Manuscript received, March 19, 1965)

\section{REFERENCES}

1. Stoney, G. J., "On the Escape of Gases from Planetary Atmospheres According to the Kinetic Theory. Part I. General Considerations," Astrophys. J. 11(4):251-258, May 1900.

2. Stoney, G. J., "On the Escape of Gases from Planetary Atmospheres According to the Kinetic Theory. Part II. More Detailed Examination," Astrophys. J. 11(5):357-372, June 1900.

3. Milne, E. A., "The Escape of Molecules from an Atmosphere, with Special Reference to the Boundary of a Gaseous Star," Cambridge Phil. Soc. Trans. 22(26):483-517, March 1923.

4. Jeans, J. H., "The Dynamical Theory of Gases," 4th rev. ed., New York: Dover Publications, Inc., 1925 (Reprinted, 1954).

5. Donahue, T. M., and Thomas, G., "Distribution of Hydrogen in the Outer Atmosphere," Planet. Space Sci. 10:65-72, 1963. 
6. Lennard-Jones, J. E., "Free Paths in a Non-Uniform Rarefied Gas with an Application to the Escape of Molecules from Isothermal Atmospheres," Cambridge Phil. Soc. Trans. 22(28):535.556 , June 1923.

7. Biutner, E. K., "The Dissipation of Gas from Planetary Atmospheres. I. The Dissipation of an Isothermal Ideal Gas in a Central Field of Gravitation," Astron. Zh. 35(4):572-582, JulyAugust 1958; Translation in: Soviet Astron. AJ 2(4):528-537, July-August 1958.

8. Biutner, E. K., "Gas Dissipation from Planetary Atmospheres. II. The Total Velocity of Gas Dissipation from a Planetary Atmosphere: the Problem of Terrestrial Helium," Astron. Zh. 36(1):89-99, January-February 1959; Translation in: Soviet Astron. AJ 3(1):92-102, JanuaryFebruary 1959.

9. Chamberlain, J. W., "Planetary Coronae and Atmospheric Evaporation," Planet. Space Sci. 11(8):901-960, August 1963.

10. Singer, S. F., "Does the Earth Possess a Hydrogen Tail?", Am. Geophys. Union Trans. 44(1):84-85, March 1963 (Abstract).

11. Öpik, E. J., and Singer, S. F., "Distribution of Density in a Planetary Exosphere. II," Phys. Fluids 4(2):221-233, February 1961.

12. Liwshitz, M., "The Distribution of Hydrogen in the Earth's Thermosphere," University of Maryland Technical Report (in press) 1965.

13. Chapman, S., and Cowling, T. G., "The Mathematical Theory of Non-Uniform Gases," 2nd ed., Cambridge: University Press, 1952.

14. Kockarts, G., and Nicolet, M., "The Aeronomical Problem of Neutral Helium and Hydrogen," Ann. Geophys. 18(3):269-290, 1962 (In French).

15. Herring, J., and Kyle, L., "Density in a Planetary Exosphere," J. Geophys. Res. 66(6):19801982, June 1961.

16. Amodt, R. E., and Case, K. M., "Density in Simple Model of the Exosphere," Phys. Fluids 5(9): 1019-1021, September 1962.

17. Kastner, S. O., "Hydrogen Diffusion in the Thermosphere by Monte Carlo Method," J. Geophys. Res. 69(1):157-174, January 1, 1964; "Correction," J. Geophys. Res. 69(13):2872, July 1, 1964.

18. Cashwell, E. D., and Everett, C. J., "Practical Manual on the Monte Carlo Method for Random Walk Problems," New York: Pergamon Press, 1959.

19. Chung, K. L., "Markov Chains with Stationary Transition Probabilities," Berlin: SpringerVerlag, 1960.

20. Takacs, L., "Stochastic Processes. Problems and Solutions," London: Methuen and Co., 1960-. 
21. Mason, E. A., Vanderslice, J. T., and Yos, J. M., "Transport Properties of High-Temperature Multicomponent Gas Mixtures," Phys. Fluids 2(6):688-694, November-December 1959.

22. Krupenie, P. H., Mason, E. A., and Vanderslice, J. T., "Interaction Energies and Transport Coefficients of $\mathrm{Li}+\mathrm{H}$ and $\mathrm{O}+\mathrm{H}$ Gas Mixtures at High Temperatures," J. Chem. Phys. 39(10):2399-2408, November 15, 1963.

23. Champion, K. S. W., and Minzner, R. A., "Revision of United States Standard Atmosphere 90 to 700 kilometers," Rev. Geophys. 1(1):57-84, February 1963.

24. Harris, I., and Priester, W., "Theoretical Models for the Solar-Cycle Variation of the Upper Atmosphere," NASA TN D-1444, August 1962.

25. Fisz, M., "Probability Theory and Mathematical Statistics," 3rd ed., New York: John Wiley and Sons, Inc., 1963.

26. Bates, D. R., and Patterson, T. N. L., "Hydrogen Atoms and Ions in the Thermosphere and Exosphere," Planet. Space Sci. 5(4):257-273, August 1961.

27. Hanson, W. B., and Patterson, T. N. L., "Diurnal Variation of the Hydrogen Concentration in the Exosphere," Planet. Space Sci. 11(9):1035-1052, September 1963.

28. Donahue, T. M., and McAfee, J. R., "Influence of Lateral Flow on the Diurnal Variation in Exospheric Hydrogen," Planet. Space Sci. 12(11):1045-1054, November 1964.

29. Harris, I., and Priester, W., Report on the Atmospheric Structure in the Region from 120 to $800 \mathrm{~km}$, to be presented to the COSPAR Meeting, Buenos Aires, May 1965 (preprint). 


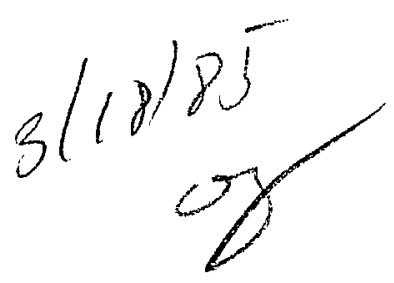

"The deronautical and space activities of the United States shall be conducted so as to contribute. . . to the expansion of buman knowledge of phenomend in the atmospbere and space. The Administration shall provide for the widest practicable and appropriate dissemination of information concerning its activities and the results thereof."

-National Aeronautics and Space Act of 1958

\section{NASA SCIENTIFIC AND TECHNICAL PUBLICATIONS}

TECHNICAL REPORTS: Scientific and technical information considered important, complete, and a lasting contribution to existing knowledge.

TECHNICAL NOTES: Information less broad in scope but nevertheless of importance as a contribution to existing knowledge.

TECHNICAL MEMORANDUMS: Information receiving limited distribution because of preliminary data, security classification, or other reasons.

CONTRACTOR REPORTS: Technical information generated in connection with a NASA contract or grant and released under NASA auspices.

TECHNICAL TRANSLATIONS: Information published in a foreign language considered to merit NASA distribution in English.

TECHNICAL REPRINTS: Information derived from NASA activities and initially published in the form of journal articles.

SPECIAL PUBLICATIONS: Information derived from or of value to NASA activities but not necessarily reporting the results of individual NASA-programmed scientific efforts. Publications include conference proceedings, monographs, data compilations, handbooks, sourcebooks, and special bibliographies.

Details on the availability of these publications may be obtained from:

SCIENTIFIC AND TECHNICAL INFORMATION DIVISION

NATIONAL AERONAUTICS AND SPACE ADMINISTRATION

Washington, D.C. 20546 